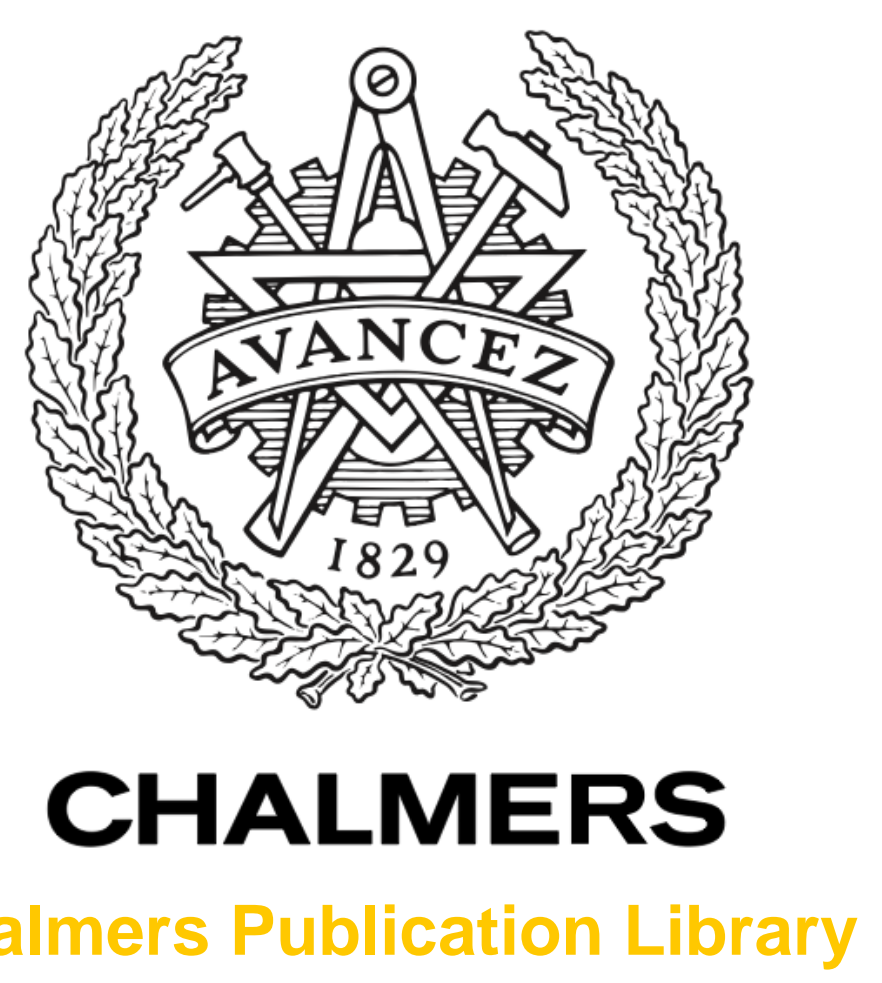

Chalmers Publication Library

Impact of Damping on Large Signal VCSEL Dynamics

This document has been downloaded from Chalmers Publication Library (CPL). It is the author's version of a work that was accepted for publication in:

24th IEEE International Semiconductor Laser Conference (ISLC), 7-10 Sept. 2014 (ISSN:

0899-9406)

Citation for the published paper:

Haglund, E. ; Westbergh, P. ; Gustavsson, J. et al. (2014) "Impact of Damping on Large Signal VCSEL Dynamics". 24th IEEE International Semiconductor Laser Conference

(ISLC), 7-10 Sept. 2014 pp. 78-79.

http://dx.doi.org/10.1109/ISLC.2014.172

Downloaded from: http://publications.lib.chalmers.se/publication/206909

Notice: Changes introduced as a result of publishing processes such as copy-editing and formatting may not be reflected in this document. For a definitive version of this work, please refer to the published source. Please note that access to the published version might require a subscription.

Chalmers Publication Library (CPL) offers the possibility of retrieving research publications produced at Chalmers University of Technology. It covers all types of publications: articles, dissertations, licentiate theses, masters theses, conference papers, reports etc. Since 2006 it is the official tool for Chalmers official publication statistics. To ensure that Chalmers research results are disseminated as widely as possible, an Open Access Policy has been adopted.

The CPL service is administrated and maintained by Chalmers Library. 


\title{
Impact of Damping on Large Signal VCSEL Dynamics
}

\author{
Emanuel P. Haglund, Petter Westbergh, Johan S. Gustavsson, and Anders Larsson \\ Photonics Laboratory, Department of Microtechnology and Nanoscience (MC2) \\ Chalmers University of Technology, Göteborg, Sweden. E-mail: emanuel.haglund@chalmers.se
}

\begin{abstract}
The dependence of large signal VCSEL dynamics on damping is studied through time-domain measurements of turn-on transients and timing jitter for VCSELs having $K$-factors from 0.1 to $0.4 \mathrm{~ns}$.
\end{abstract}

\section{INTRODUCTION}

The increasing demand for high-speed optical interconnects has resulted in large efforts to increase the speed of the $850 \mathrm{~nm}$ oxide confined VCSEL. We have previously shown that a significant increase of the small signal modulation bandwidth can be achieved by reducing the damping of the modulation response [1]. However, damping also has an effect on the large signal dynamics and proper damping is needed for high quality optical eyes. While low damping offers the high bandwidth and fast rise time needed for very high speed modulation, it gives rise to higher transient overshoot and more data dependent timing jitter, resulting in horizontal eye closure. Higher damping reduces overshoot and jitter but too much damping results in insufficient bandwidth and vertical eye closure. The optimum amount of damping therefore depends on the bit rate, with less damping expected to be required at higher bit rates.

Here we present results from a study of the dependence of large signal VCSEL dynamics on damping with the $K$-factor (which quantifies the increase of damping with resonance frequency) varied from 0.1 to $0.4 \mathrm{~ns}$.

\section{DESIGN AND BASIC PERFORMANCE}

The VCSELs used in this study have been described in detail elsewhere [2]. Strained quantum wells, low resistance and low loss distributed Bragg reflectors (DBRs), and multiple oxide apertures are employed to enable high speed modulation. The size of the oxide apertures closest to the active region is $9 \mu \mathrm{m}$. Damping was varied by varying the photon lifetime from $\sim 2$ to $6 \mathrm{ps}$ (VCSELs A to D). This was achieved by shallow surface etching to modify the top DBR reflectivity of individual VCSELs [2].

The power-current characteristics (Fig. 1) show that both threshold current and slope efficiency increases with reduced photon lifetime. Increased slope efficiency is expected to increase the vertical eye opening under binary large signal modulation.
The small signal modulation response for VCSELs having the shortest and longest photon lifetime (least and most damped) is shown in Fig. 2. Extracted $K$ and $D$-factors for VCSELs A-D range from 0.1 to $0.4 \mathrm{~ns}$ and 4.9 to $6.4 \mathrm{GHz} / \mathrm{mA}^{-1 / 2}$, respectively (Fig. 3). The modulation bandwidth has a maximum value of $21 \mathrm{GHz}$ at $K=0.2 \mathrm{~ns}$ (VCSEL B). Lower and higher $K$ results in smaller bandwidth due to reduced resonance frequency and excessive damping, respectively [2].

\section{LARGE SIGNAL DYNAMICS}

Turn-on transients were studied by applying a voltage step generated by an SHF 12103A bit pattern generator programmed with repeated series of 16 one's and 16 zero's at a bit rate of $10 \mathrm{Gbps}$ (1.6 ns pulse length). Optical waveforms were recorded by a $30 \mathrm{GHz}$ detector (VI Systems D30-850M) connected to an $70 \mathrm{GHz}$ equivalent time sampling oscilloscope (Agilent Infiniium DCA-J $86100 \mathrm{C})$. The recorded waveforms at a bias current of $9 \mathrm{~mA}$, along with extracted rise times, overshoots and signal amplitudes, are shown in Fig. 4. Although the measured rise times were largely limited by the bandwidth of the measurement system it is clear that the rise time is reduced with reduced damping. In addition, the signal amplitude increases with reduced damping. This contributes to a larger vertical eye opening. However, the recorded waveforms also show that the overshoot and the settling time for the relaxation oscillation increase with reduced damping. This gives rise to data dependent timing jitter when the bit period is shorter than the settling time.

Total jitter (TJ) was measured by detecting the optical signal using a linear $22 \mathrm{GHz}$ photoreceiver (New Focus 1484-A-50) connected to an error analyzer (SHF 11100B). The linear receiver was used to study the intrinsic behaviour of the VCSELs. The sampling instant was swept across the transition region to obtain bathtub curves which were fitted to the Dual-Dirac model. The TJ is the width of the transition region at $\mathrm{BER}=10^{-12}$ in the extrapolation from the fit. The TJ at 10 and $25 \mathrm{Gbps}$ PRBS $2^{7}-1$ versus $K$ at $9 \mathrm{~mA}$ bias current, together with the corresponding eye diagrams at $25 \mathrm{Gbps}$, is shown in Fig. 5. Clearly, reduced damping increases the TJ which results in horizontal eye closure. 


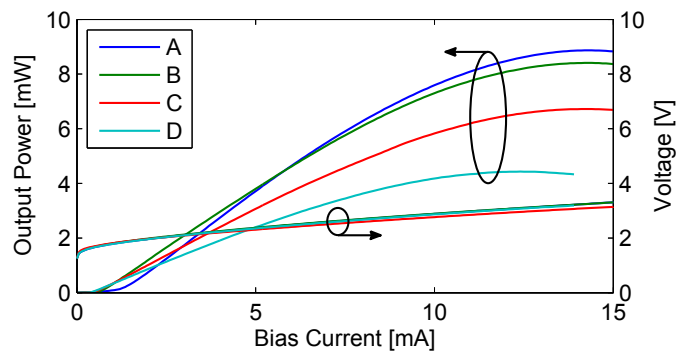

Fig. 1. Optical output power and voltage vs. current for VCSELs A-D with increasing photon lifetime.

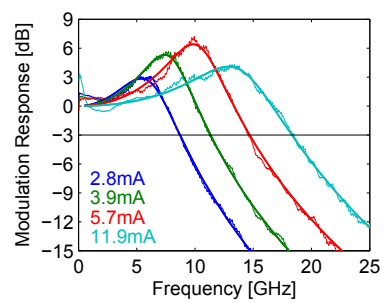

(a)

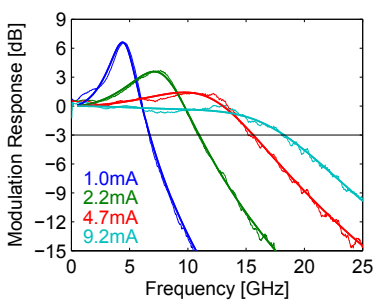

(b)
Fig. 2. Small signal modulation response for (a) VCSEL A and (b) D.

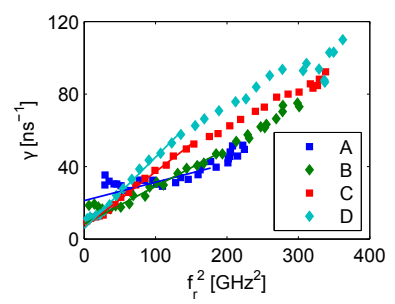

(a)

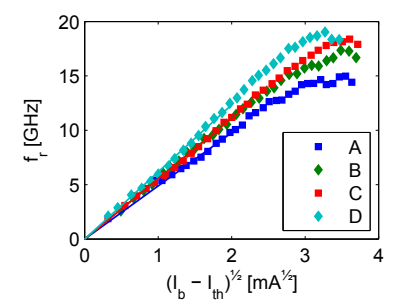

(b)
Fig. 3. (a) Damping rate vs. resonance frequency squared. Fits used to extract the $K$-factors are shown. (b) Resonance frequency vs. square root of bias current above threshold. Fits used to extract the $D$-factors are shown.

At the lower bit rate the $\mathrm{TJ}$ is smaller since the bit period approaches the settling time for the relaxation oscillations.

\section{CONCLUSION}

We have shown that damping has a significant effect on the large signal dynamics of VCSELs. Low damping, which is needed at very high data rates, enables a fast rise time and a large optical signal amplitude but gives rise to a larger overshoot and a longer settling time causing more timing jitter. Lower data rates can afford more damping for high quality optical eyes.

\section{ACKNOWLEDGMENT}

This project is financially supported by the Swedish Foundation for Strategic Research.

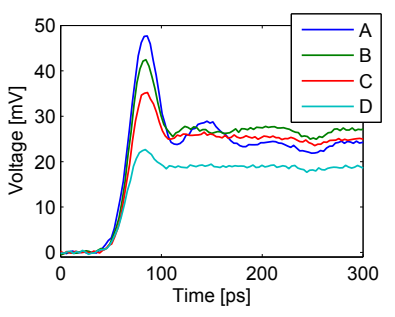

(a)

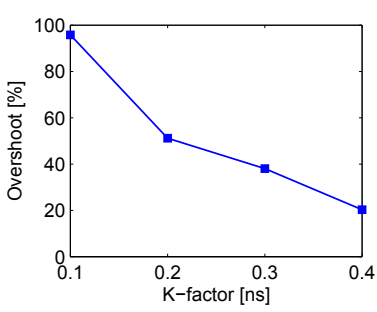

(c)

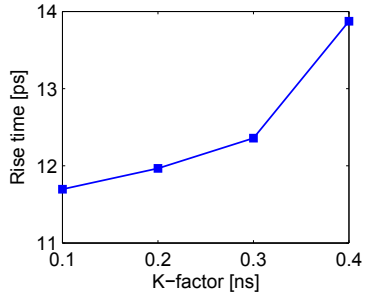

(b)

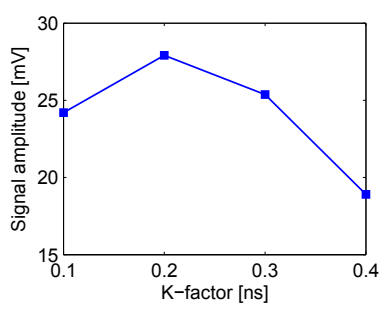

(d)
Fig. 4. (a) Step response for VCSELs A-D. (b-c) Rise time, overshoot and signal amplitude at $9 \mathrm{~mA}$ vs. $K$, respectively.

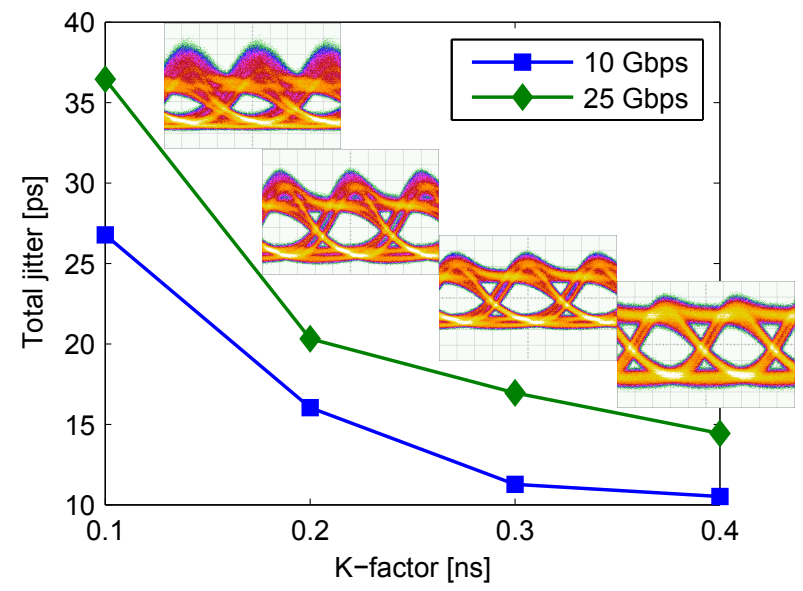

Fig. 5. Total jitter vs. $K$ at $9 \mathrm{~mA}$. Insets: Eye diagrams at $25 \mathrm{Gbps}$ (vertical scale: $60,50,50$ and $25 \mathrm{mV} / \mathrm{div}$ ).

\section{REFERENCES}

[1] P. Westbergh, J. Gustavsson, B. Kögel, A. Haglund, A. Larsson, and A. Joel, "Speed enhancement of VCSELs by photon lifetime reduction," Electronics Letters, vol. 46, no. 13, p. 938, 2010. [Online]. Available: http://digital-library.theiet.org/content/ journals/10.1049/el.2010.0779

[2] P. Westbergh, J. S. Gustavsson, B. Kögel, A. Haglund, and A. Larsson, "Impact of Photon Lifetime on HighSpeed VCSEL Performance," IEEE Journal of Selected Topics in Quantum Electronics, vol. 17, no. 6, pp. 1603-1613, Nov. 2011. [Online]. Available: http://ieeexplore.ieee.org/lpdocs/epic03/ wrapper.htm?arnumber $=5740303$ 